\title{
Identification of Parental Chromosomes in Sexual Intergeneric Hybrid Progenies between Citrus Cultivar 'Nanpu' Tangor and Citropsis schweinfurthii in the
} Subfamily Aurantioideae

\author{
Kichi Yasuda $^{1}$, Masaki Yahata ${ }^{2}$, Masayoshi Shigyo ${ }^{3}$, Ryoji Matsumoto ${ }^{4}$, Tsutomu Yabuya ${ }^{5}$ \\ and Hisato Kunitake ${ }^{5 *}$
}

${ }^{1}$ The Interdisciplinary Graduate School of Agriculture and Engineering, University of Miyazaki, Miyazaki 889-2192, Japan

${ }^{2}$ The Faculty of Agriculture, Shizuoka University, Shizuoka 422-8529, Japan

${ }^{3}$ Faculty of Agriculture, Yamaguchi University, Yamaguchi 753-8515, Japan

${ }^{4}$ The Faculty of Agriculture, Saga University, Saga 840-8502, Japan

${ }^{5}$ Faculty of Agriculture, University of Miyazaki, Miyazaki 889-2192, Japan

To obtain cytogenetic information on sexual intergeneric hybrid progenies between Citropsis schweinfurthii and 'Nanpu' tangor (Citrus spp.), we conducted Chromomycin $\mathrm{A}_{3}$ (CMA) chromosome banding and genomic in situ hybridization (GISH) analyses. The CMA karyotype composition of $C$. schweinfurthii was very simple (14D+4E), while that of 'Nanpu' tangor showed more complicated chromosome types $(1 \mathrm{~A}+4 \mathrm{C}+5 \mathrm{D}+8 \mathrm{E})$. Two progenies with $C$. schweinfurthii as the maternal parent showed a karyotype of $1 \mathrm{~A}+1 \mathrm{C}+11 \mathrm{D}+5 \mathrm{E}$, suggesting that each of the type $A$ and $C$ chromosomes was inherited from 'Nanpu' tangor. GISH-CMA analysis in one of these progenies showed a clear distinction between parental chromosomes, and revealed that the hybrid progeny arose due to fertilization between a female gamete with a CMA karyotype composition of 7D+2E from $C$. schweinfurthii and a male $1 \mathrm{~A}+1 \mathrm{C}+4 \mathrm{D}+3 \mathrm{E}$ gamete from 'Nanpu' tangor. The result suggests that $C$. schweinfurthii had homomorphic homologous chromosomes as a wild species, while 'Nanpu' tangor had heteromorphic homologous chromosomes.

Key Words: Chromomycin $\mathrm{A}_{3}$ (CMA) karyotype, cross incompatibility, genomic in situ hybridization (GISH) analysis, GISH-CMA analysis, homologous chromosomes.

\section{Introduction}

The subfamily Aurantioideae (Citroideae) in the family Rutaceae is an important group of plants, with many species of industrial importance, including those belonging to three genera, Citrus L., Fortunella Swingle and Poncirus (L.) Raf.; therefore, it is essential for further breeding and for developing better conservation strategies to understand the phylogenetic relationships in this subfamily. Citropsis schweinfurthii (Engl.) Swing. \& M. Kell., native to Africa, is considered to be a surviving form of remote ancestors of Citrus because its leaflets very closely resemble those of Citrus in shape, texture, venation, and color (Swingle and Reece, 1967).

Received; June 5, 2009. Accepted; September 6, 2009.

This research was supported by a Grand-in-Aid for Scientific Research (C) (No. 20580034) from the Ministry of Education, Culture, Sports, Science, and Technology (Japan) and the Sasakawa Scientific Research Grant from The Japan Science Society.

* Corresponding author (E-mail: hkuni@cc.miyazaki-u.ac.jp).
In our previous study with the aim of clarifying the relationship between Citrus and Citropsis, we successfully produced seedlings for the first time by reciprocal crosses between the Citrus cultivar, 'Nanpu' tangor, and C. schweinfurthii through in vitro culture and in vitro grafting, and confirmed the hybridism by genome size and DNA analyses (Yahata et al., 2006).

The chromosome banding technique using chromomycin $\mathrm{A}_{3}$ (CMA), a guanine-cytosine (GC) base-specific fluorochrome, counterstained with adenine-thymine (AT) base-specific 4'-6-diamidino-2-phenylindole (DAPI) or distamycin A hydrochloride has been used for chromosome karyotyping in many higher plants (Davies et al., 1997; Hizume et al., 1989; Kim et al., 2002; Penas et al., 2008). In Aurantioideae, including Citrus, which has comparatively small chromosomes, CMA banding has also been employed for chromosome characterization, classification, hybridity analysis and confirming autoploidy (Befu et al., 2000; Cornelio et al., 2003; Guerra et al., 2000; Miranda et al., 1997; 
Yahata et al., 2005a, b; Yamamoto et al., 2008). Genomic in situ hybridization (GISH), a modification of fluorescence in situ hybridization (FISH), allows chromosomes from different parents, ancestors or genomes in plants to be painted in different colors (Raina and Rani, 2001). Therefore, GISH analysis has become a useful tool for the identification of parental genomes and the detection of introgression in natural allopolyploid species, somatic hybrids and sexual hybrids (Inoue et al., 2006; Kamstra et al., 1999; Lim et al., 1998; Maluszynska and Hasterok, 2005; Mukai et al., 1993; Zhou et al., 2005).

It has previously been demonstrated that hybrid progenies can be obtained by sexual hybridization between 'Nanpu' tangor and C. schweinfurthii (Yahata et al., 2006); however, little cytogenetic information on hybrid progenies has been yielded. The objective of the present study was to identify parental chromosomes in sexual intergeneric hybrid progenies between 'Nanpu' tangor and C. schweinfurthii. Cytological analysis was performed using a combination of GISH analysis and CMA chromosome banding.

\section{Materials and Methods}

\section{Plant materials}

'Nanpu' tangor \{'Kiyomi' tangor (C. unshiu Marcow. $\times$ C. sinensis Osbeck) $\times$ 'Fairchild' tangerine-tangelo [clementine (C. clementina hort. ex Tanaka) × 'Orlando' (C.paradisi Macf. $\times$ C. reticulata Blanco)]\}, C. schweinfurthii (Engl.) Swing., three progenies obtained from a cross between 'Nanpu' tangor and C. schweinfurthii ( $\mathrm{NC1}, 2$, and 3), and three progenies obtained from a cross between C. schweinfurthii and 'Nanpu' tangor (CN1, 2, and 3) were used for this study (Yahata et al., 2006). Young leaves of CN1 and CN3 after a year of acclimatization and $\mathrm{CN} 3$ grown by grafting onto C. schweinfurthii were used for CMA karyotype analysis and GISH-CMA analysis, respectively.

\section{CMA karyotype analysis}

Young leaves (approximately $3-5 \mathrm{~mm}$ long) were excised, immersed in $2 \mathrm{mM} 8$-hydroxyquinoline for $10 \mathrm{~h}$ at $4^{\circ} \mathrm{C}$ and fixed in a mixed solution of ethanol and acetic acid $(3: 1)$ for $12 \mathrm{~h}$ at $4^{\circ} \mathrm{C}$. Enzymatic maceration and air-drying were performed following the method described by Fukui (1996) with some modifications. The young leaves were washed in distilled water to remove the fixative and then macerated at $37^{\circ} \mathrm{C}$ for $40 \mathrm{~min}$ in an enzyme mixture containing $2.0 \%(\mathrm{w} / \mathrm{v})$ Cellulase Onozuka RS (Yakult Pharmaceutical Ind. Co., Ltd., Tokyo, Japan), $1.0 \%$ (w/v) Macerozyme (MP Biomedicals, Inc., Burlingame, CA, USA), 0.3\% Pectolyase Y-23 (w/v) (Kyowa Chemical Products Co., Ltd., Osaka, Japan) and $200 \mathrm{mM}$ ethylenediaminetetraacetic acid (EDTA). The chromosomes were stained with $2.0 \%$ Giemsa solution (Merck KGaA, Darmstadt, Germany) in a 1/30 phosphate buffer ( $\mathrm{pH} 6.8)$ for $30 \mathrm{~min}$. They were then rinsed with distilled water, air-dried, and observed under an optical microscope BX51 (Olympus Co., Ltd., Tokyo, Japan). After the chromosome numbers and each position were confirmed on a slide, the chromosomes were destained with both $70 \%$ and $100 \%$ ethanol. They were then stained with $0.1 \mathrm{mg} \cdot \mathrm{L}^{-1} \mathrm{CMA}$ and $0.1 \mathrm{mg} \cdot \mathrm{L}^{-1}$ Distamycin A hydrochloride (SigmaAldrich Co., Steinheim, Germany) following the method described by Befu et al. (2000) with some modifications, and observed under a fluorescence microscope BX51 (Olympus Co., Ltd.).

\section{GISH-CMA analysis}

Chromosome preparations were performed following the methods described above. GISH analysis was conducted as described by Yamashita et al. (2005) with some modifications. Total genomic DNA of C.schweinfurthii was extracted from young leaves according to the method of Doyle and Doyle (1987) with some modifications, labeled with biotin-16-dUTP using the Nick Translation Kit (Roche Diagnostics, Ltd., Rotkreuz, Switzerland), and used as the probe DNA; excess unlabeled genomic DNA of 'Nanpu' tangor was used as the blocking DNA. One hundred microliters of the hybridization mixture contained $1 \mu \mathrm{g}$ probe DNA and $20 \mu \mathrm{g}$ blocking DNA. After hybridization, probe hybridization signals were amplified with fluorescein avidin DN (Vector Laboratories Inc., Burlingame, CA, USA) and counterstained with propidium iodide (PI). The preparations were mounted with VECTA SHIELD mounting medium (Vector Laboratories Inc.) and observed under a fluorescence microscope. Photographs were taken using Fujifilm PROVIA 400F (Fujifilm Co., Ltd., Tokyo, Japan) and dual-exposed with fluoresceinisothiocyanate (FITC) and PI fluorescence. The preparations used for GISH analysis were destained with a mixed solution of ethanol and acetic acid $(3: 1)$ and $100 \%$ methanol, and air-dried. They were subsequently used for CMA banding analysis as described above.

\section{Results and Discussion}

All 6 progenies were transferred in vitro to a glass greenhouse, where they showed serious weakness in the early stages. Interestingly, progenies with 'Nanpu' tangor as the maternal parent were obviously less vigorous than those with C.schweinfurthii as the maternal parent (Fig. 1). In interspecific hybridization of other genera, such as Rhododendron and Zantedeschia, it has been noted that albinism and color-breaking in the progenies are caused by incompatibility between the plastome and nuclear genome (Ureshino et al., 1999; Yao et al., 1994). The intergeneric hybrid progenies used in the present study had a plastome from the maternal parent and a nuclear genome from both parents (Yahata et al., 2006), suggesting that the difference in growth observed in these reciprocal progenies may also have been caused by incompatibility between the plastome from 'Nanpu' 


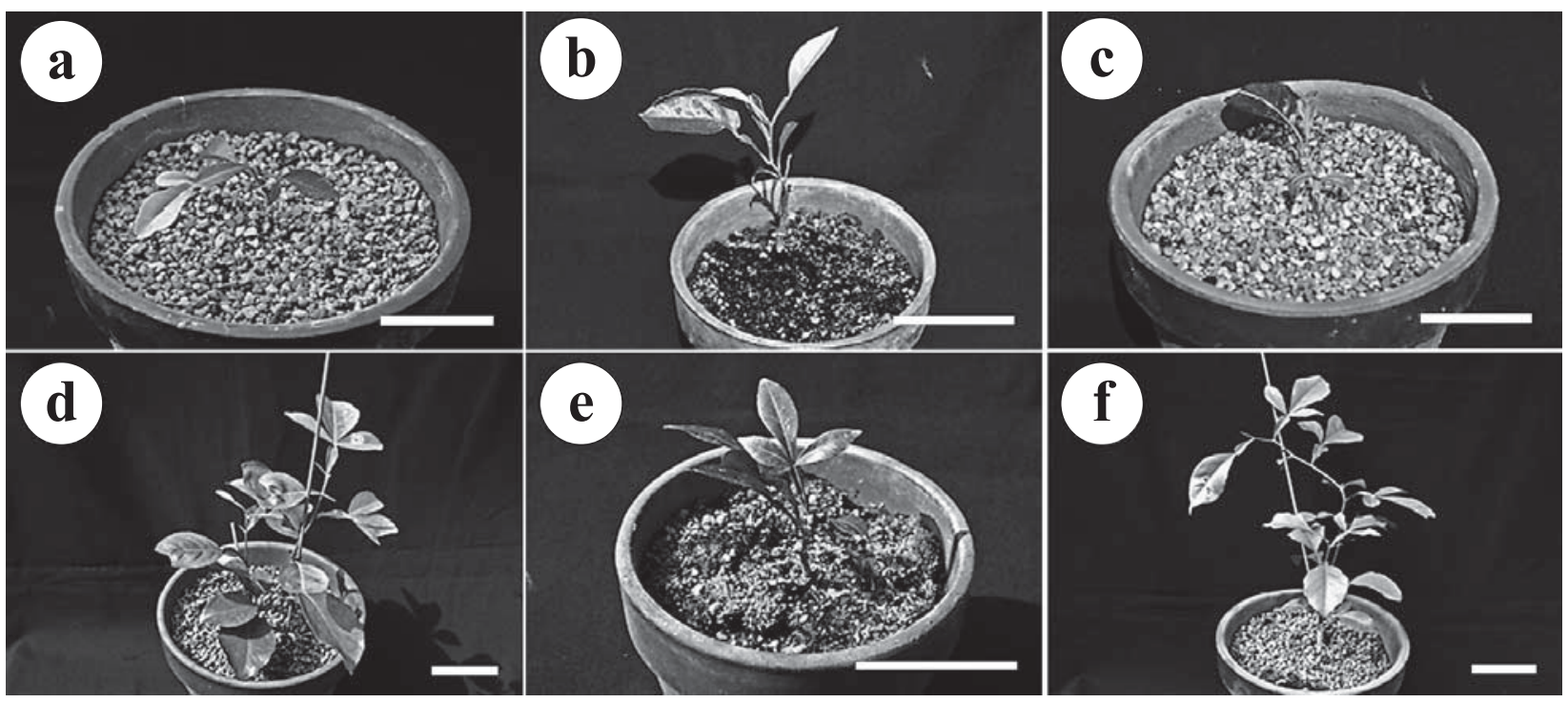

Fig. 1. Intergeneric hybrids obtained from reciprocal crosses between C. schweinfurthii and 'Nanpu' tangor a year after acclimatization. Bars = $5 \mathrm{~cm}$. a-c: $\mathrm{NC1}, \mathrm{NC2}$, and $\mathrm{NC} 3$ obtained from a cross between 'Nanpu' tangor and C. schweinfurthii d-f: CN1, CN2, and CN3 obtained from a cross between C. schweinfurthii and 'Nanpu' tangor.

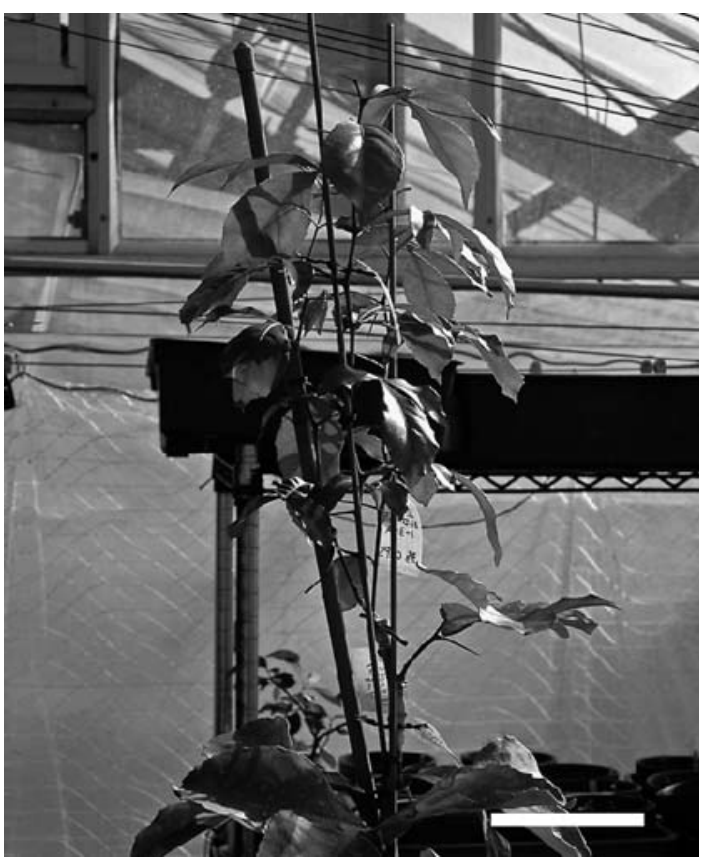

Fig. 2. The intergeneric hybrid $(\mathrm{CN} 3)$ showed vigorous growth a year after grafting onto C. schweinfurthii. Bar $=10 \mathrm{~cm}$.

tangor and the nuclear genome from C. schweinfurthii.

Three years after acclimatization, all progenies with C. schweinfurthii as the maternal parent had maintained the status shown in Figure 1, while all progenies with 'Nanpu' tangor as the maternal parent had died. When 6 intergeneric hybrid progenies (NC1-3 and CN1-3) were grafted onto C.schweinfurthii, only $\mathrm{CN} 3$ showed vigorous growth (Fig. 2). The young leaves obtained from both $\mathrm{CN} 1$ and $\mathrm{CN} 3$ were suitable for chromosome analyses, though the leaves of $\mathrm{CN} 3$ were in better condition than those of CN1. Thus, the leaves of both
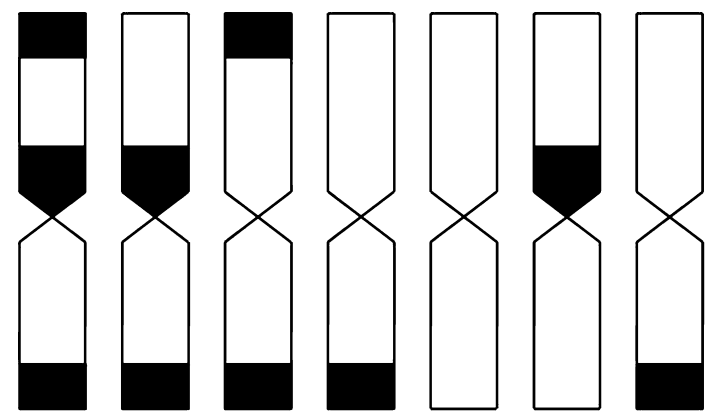

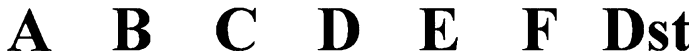

Fig. 3. Idiograms of CMA banding pattern according to Yamamoto and Tominaga (2003) and Yamamoto et al. (2007). A = two telomeric bands and one proximal band, $\mathrm{B}=$ one telomeric and one proximal band, $\mathrm{C}=$ two telomeric bands, $\mathrm{D}=$ one telomeric, $\mathrm{E}=$ no band, $\mathrm{F}=$ one proximal band, and $\mathrm{Dst}=$ type $\mathrm{D}$ with a satellite chromosome.

CN1 and CN3 were used for CMA banding analysis, and only those of $\mathrm{CN} 3$ were used for further GISH-CMA analysis.

The chromosomes were classified into the following 7 types based on the number and position of the CMA banding pattern according to Yamamoto and Tominaga (2003) and Yamamoto et al. (2007): $A=2$ telomeric bands and 1 proximal band; $\mathrm{B}=1$ telomeric and 1 proximal band; $\mathrm{C}=2$ telomeric bands; $\mathrm{D}=1$ telomeric band; $\mathrm{E}=$ no bands; $\mathrm{F}=1$ proximal band; and $\mathrm{Dst}=$ type D with a satellite chromosome (Fig. 3). The CMA karyotype compositions of the parents were $14 \mathrm{D}+4 \mathrm{E}$ in C. schweinfurthii and $1 \mathrm{~A}+4 \mathrm{C}+5 \mathrm{D}+8 \mathrm{E}$ in 'Nanpu' tangor, respectively (Fig. 4). C. schweinfurthii had a very 
simple karyotype composition with only type D and $\mathrm{E}$ chromosomes, which coincided with the result of a previous study (Yamamoto et al., 2008). In addition to these types, 'Nanpu' tangor, in contrast, had type A and $\mathrm{C}$ chromosomes. Both progenies $(\mathrm{CN} 1$ and $\mathrm{CN} 3)$ showed a karyotype of $1 \mathrm{~A}+1 \mathrm{C}+11 \mathrm{D}+5 \mathrm{E}$, suggesting that each one of the type $\mathrm{A}$ and $\mathrm{C}$ chromosomes were inherited from 'Nanpu' tangor. To gather further cytogenetic information about the distribution of the CMA karyotypes of both parents, we then performed GISHCMA analysis.
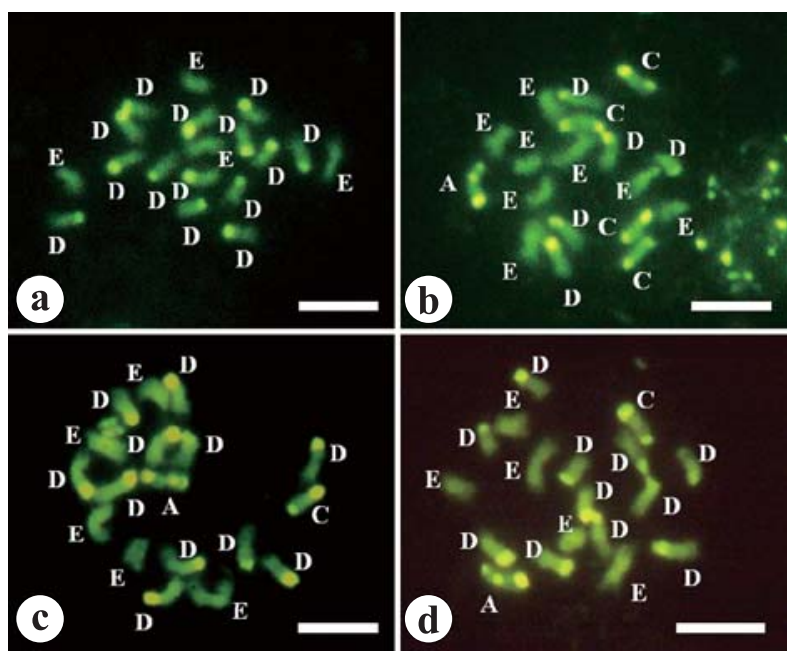

Fig. 4. Photographs of CMA banding patterns in somatic chromosomes of intergeneric hybrids obtained from a cross between C. schweinfurthii and 'Nanpu' tangor, and its parents. Bars = $5 \mu \mathrm{m}$. a: C. schweinfurthii, b: 'Nanpu' tangor, c: CN1, d: CN3.

\section{C. schweinfurthii}

14D + 4E

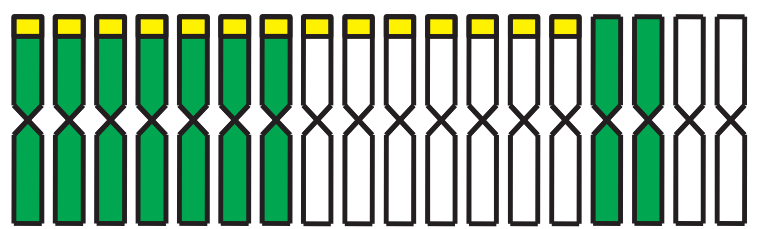

Female gamete $7 \mathrm{D}+2 \mathrm{E}$

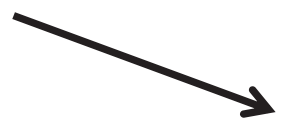

While GISH analysis showed a clear distinction between parental chromosomes in CN3 (Fig. 5), some centromeric regions of 'Nanpu' tangor showed small green signals by C. schweinfurthii genomic probe. Matsuyama et al. (1996, 1999) and Moraes et al. (2007) reported rDNA sites and a large number of simple sequence repeats in the telomeric and centromeric regions of Citrus. Moraes et al. (2007) also revealed that type A chromosomes of some grapefruits (C.paradise Macfad.) and pummelos [C. maxima (Burm.) Merr.] have 45S rDNA sites near the centromere. In the present study, a small green signal was detected on the type A chromosome of 'Nanpu' tangor, suggesting that the 45S rDNA site of $C$. schweinfurthii might hybridize with that of 'Nanpu' tangor due to their extremely high homology.

Only a few GISH studies on the genome analysis of somatic hybrids and chromosome karyotyping in

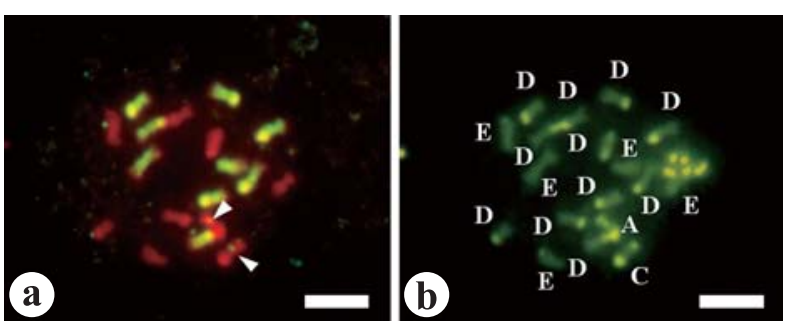

Fig. 5. GISH-CMA analysis of somatic chromosomes at metaphase in young leaves of hybrids between C.schweinfurthii and 'Nanpu' tangor (CN3). Bars $=5.0 \mu \mathrm{m}$. a: GISH analysis with C. schweinfurthii (Green) genomic probe, b: CMA staining. Arrowheads showed small green signals by C. schweinfurthii genomic probe in centromeric regions of 'Nanpu' tangor.

\section{'Nanpu' tangor}

$1 A+4 C+5 D+8 E$

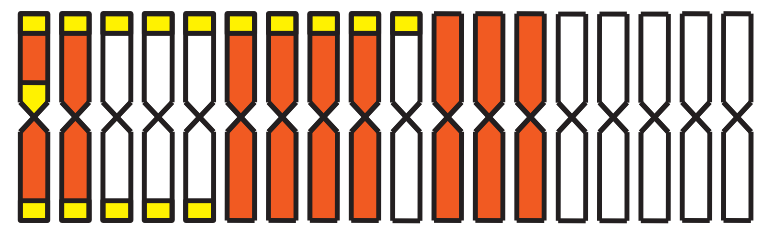

Male gamete $\quad 1 \mathrm{~A}+1 \mathrm{C}+4 \mathrm{D}+3$

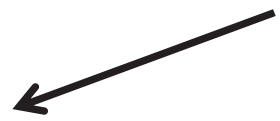

C. schweinfurthii $\times$ 'Nanpu' tangor (CN3)

$1 \mathrm{~A}+1 \mathrm{C}+11 \mathrm{D}+\mathbf{5 E}$

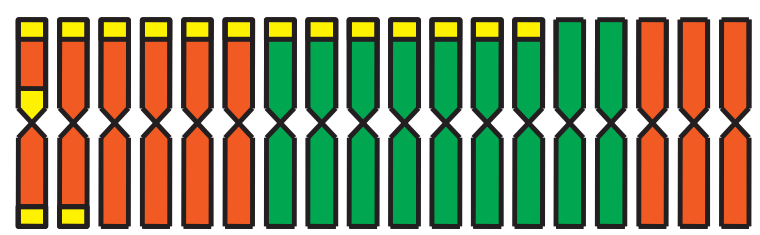

Fig. 6. Idiograms of GISH-CMA analysis of somatic chromosomes at metaphase in young leaves of hybrids between C. schweinfurthii and 'Nanpu' tangor (CN3). C. schweinfurthii genome (Green) and 'Nanpu' tangor genome (Red) in GISH and CMA bands (Yellow). 
Aurantioideae have been reported (Fu et al., 2004; Kitajima et al., 2007; Preedasuttijit et al., 2007). To the best of our knowledge, the present study is the first report of its application to sexual intergeneric hybrids of Aurantioideae. The chromosomes of $\mathrm{CN} 3$ used for GISH analysis were destained and then stained by CMA. GISH-CMA revealed that $\mathrm{CN} 3$ had $7 \mathrm{D}+2 \mathrm{E}$ chromosomes derived from C. schweinfurthii and $1 \mathrm{~A}+1 \mathrm{C}+4 \mathrm{D}$ $+3 \mathrm{E}$ chromosomes derived from 'Nanpu' tangor, indicating that $\mathrm{CN} 3$ arose from fertilization between female and male gametes with these CMA karyotype compositions (Fig. 6). Interestingly, just half of type D and $\mathrm{E}$ chromosomes of $C$. schweinfurthii was inherited in CN3; however, the chromosomes of 'Nanpu' tangor showed a different mode of inheritance, for example, only one out of 4 type $\mathrm{C}$ chromosomes was inherited in CN3. These results suggest that chromosome pairing between homologous chromosomes with different CMA karyotypes occurred in the meiosis of 'Nanpu' tangor. The existence of heteromorphic homologous chromosomes was generically observed on previous CMA banding analysis in Citrus spp. (Befu et al., 2001; Cornelio et al., 2003; Yamamoto et al., 2007). Yang et al. (2002) proved that two pummelo cultivars had heteromorphic homologous chromosomes based on the number of CMA karyotypes of their intraspecific hybrids. Thus, the reason for the different mode of chromosome pairing between C.schweinfurthii and 'Nanpu' tangor in the present study might include that C. schweinfurthii has homomorphic homologous chromosomes as a wild species, while 'Nanpu' tangor has heteromorphic homologous chromosomes because it is a horticultural cultivar derived from several crossings with some interspecific cross combinations.

In the present study, we successfully identified the parental genomes and their CMA karyotype composition in intergeneric hybrid progenies between C. schweinfurthii and 'Nanpu' tangor using GISH-CMA analysis. We expect to obtain further information on the relationship between Citrus and Citropsis by investigating meiosis and through the GISH-CMA observation of interchromosomal recombination in backcrossed progenies if these progenies flower.

\section{Acknowledgments}

The authors are grateful to Dr. Masahiro Mii of the Graduate School of Horticulture, Chiba University, for his advice and critical reading of this manuscript. Thanks are due to Dr. Koichi Inoue of the United Graduate School of Agricultural Sciences, Kagoshima University, for providing advice on the methods of GISH analysis. We also thank Mr. Yukiyoshi Katayama of the Faculty of Agriculture, Saga University, and Mr. Yasuhiro Okuno for kindly providing the experimental materials.

\section{Literature Cited}

Befu, M., A. Kitajima, Y. X. Ling and K. Hasegawa. 2000.
Classification of 'Tosa-Buntan' pummelo (Citrus grandis [L.] Osb.), 'Washington' navel orange (C. sinensis [L.] Osb.) and trifoliate orange (Poncirus trifoliata [L.] Raf.) chromosomes using young leaves. J. Japan. Soc. Hort. Sci. 69: 22-28.

Befu, M., A. Kitajima and K. Hasegawa. 2001. Chromosome composition of some citrus species and cultivars based on the chromomycin $\mathrm{A}_{3}$ (CMA) banding patterns. J. Japan. Soc. Hort. Sci. 70: 83-88 (In Japanese with English abstract).

Cornelio, M. T. M. N., A. R. S. Figueiroa, K. G. B. Santos, R. Carvalho, W. S. Soares Filho and M. Guerra. 2003. Chromosomal relationships among cultivars of Citrus reticulata Blanco, its hybrids and related species. Plant Syst. Evol. 240: 149-161.

Davies, B. J., I. E. W. O'Brien and B. G. Murray. 1997. Karyotypes, chromosome bands and genome size variation in New Zealand endemic gymnosperms. Plant Syst. Evol. 208: 169-185.

Doyle, J. and J. L. Doyle. 1987. A rapid DNA isolation procedure for small quantities fresh leaf tissue. Phytochem. Bull. 19: $11-15$.

Fu, C. H., C. L. Chen, W. W. Guo and X. X. Deng. 2004. GISH, AFLP and PCR-RFLP analysis of an intergeneric somatic hybrid combining Goutou sour orange and Poncirus trifoliata. Plant Cell Rep. 23: 391-396.

Fukui, K. 1996. Plant chromosome at mitosis. p. 1-17. In: K. Fukui and S. Nakayama (eds.). Plant chromosome. Laboratry methods. CRC Press, Boca Raton.

Guerra, M., K. G. B. Santos, A. E. B. Silva and F. Ehrendorfer. 2000. Heterochromatin banding patterns in RutaceaeAurantioideae - a case of parallel chromosomal evolution. Amer. J. Bot. 87: 735-747.

Hizume, M., A. Ohgiku and A. Tanaka. 1989. Chromosome banding in the genus Pinus II. Interspecific variation of fluorescent banding patterns in $P$. densiflora and $P$. thunbergii. Bot. Mag. (Tokyo) 102: 25-36.

Inoue, K., T. Kato, A. Nobukuni, H. Kunitake and T. Yabuya. 2006. Characterization of tetraploid plants regenerated via protoplast culture of Iris fulva and their crossability with Japanese irises. Sci. Hortic. 110: 334-339.

Kamstra, S. A., A. G. J. Kuipers, M. J. D. Jeu, M. S. Ramanna and E. Jacbsen. 1999. The extent and position of homoeologous recombination in a distant hybrid of Alstroemeria: a molocular cytogenetic assessment of first generation backcross progenies. Chromosoma 108: 52-63.

Kim, E. S., E. O. Punina and A. V. Rodionov. 2002. Chromosome CPD (PI/DAPI)- and CMA/DAPI-banding patterns in Allium cepa L.. Russ. J. Genet. 38: 392-398.

Kitajima, A., A. Yamasaki, T. Habu, B. Preedasuttijit and K. Hasegawa. 2007. Chromosome identification and karyotyping of satsuma mandarin by genomic in situ hybridization. J. Amer. Soc. Hort. Sci. 132: 836-841.

Lim, K. Y., I. J. Leitch and A. R. Leitch. 1998. Genomic characterization and the detection of raspberry chromatin in polyploidy Rubus. Theor. Appl. Genet. 97: 1027-1033.

Maluszynska, A. and R. Hasterok. 2005. Identification of individual chromosomes and parental genomes in Brassica juncea using GISH and FISH. Cytogenet. Genome Res. 109: 310-314.

Matsuyama, T., T. Akihama, Y. Ito, M. Omura and K. Fukui. 1996. Characterization of heterochromatic regions in 'Trovita' orange (Citrus sinensis Osbeck) chromosomes by the fluorescent staining and FISH methods. Genome 39: 941-945.

Matsuyama, T., T. Akihama, Y. Ito, M. Omura and K. Fukui. 1999. Distribution of TGG repeat-related sequences in 'Trovita' orange (Citrus sinensis Osbeck) chromosomes. Genome 42: $1251-1254$. 
Miranda, M., F. Ikeda, T. Endo, T. Moriguchi and M. Omura. 1997. Comparative analysis on the distribution of heterochromatin in Citrus, Poncirus and Fortunella chromosomes. Chromosome Res. 5: 86-92.

Moraes, A. P., W. S. S. Filho and M. Guerra. 2007. Karyotype diversity and the origin of grapefruit. Chromosome Res. 15: $115-121$.

Mukai, Y., Y. Nakahara and M. Yamamoto. 1993. Simultaneous discrimination of the three genomes in hexaploid wheat by multicolor fluorescence in situ hybridization using total genomic and highly repeated DNA probes. Genome 36: 489494.

Penas, M. L. L., G. Bernardello and R. Kiesling. 2008. Karyotypes and fluorescent chromosome banding in Pyrrhocactus (Cactaceae). Plant Syst. Evol. 272: 211-222.

Preedasuttijit, B., A. Kitajima, A. Yamasaki, T. Ogata and K. Hasegawa. 2007. Chromosome identification and characterization in trifoliate orange (Poncirus trifoliata (L.) Raf.) by CMA and PI/DAPI staining and GISH. J. Japan. Soc. Hort. Sci. 76: 197-204.

Raina, S. N. and V. Rani. 2001. GISH technology in plant genome research. Methods Cell Sci. 23: 83-104.

Swingle, W. T. and P. C. Reece. 1967. The botany of Citrus and its wild relatives in the orange subfamily. p. 190-430. In: W. Reuther, H. J. Webber and L. D. Bachelor (eds.). The citrus industry, Vol. 1. University of California, Berkeley.

Ureshino, K., I. Miyajima, Y. Ozaki, N. Kobayashi, A. Michishita and M. Akabane. 1999. Appearance of albino seedling and ptDNA inheritance in interspecific hybrids of azalea. Euphytica 110: 61-66.

Yahata, M., H. Kunitake, T. Yabuya, K. Yamashita, Y. Kashihara and H. Komatsu. 2005a. Production of a doubled haploid from a haploid pummelo using colchicine treatment of axillary shoot buds. J. Amer. Soc. Hort. Sci. 130: 899-903.
Yahata, M., H. Kunitake, K. Yasuda, K. Yamashita, H. Komatsu and R. Matsumoto. 2006. Production of sexual hybrid progenies for clarifying the phylogenic relationship between Citrus and Citropsis species. J. Amer. Soc. Hort. Sci. 131: 764-769.

Yahata, M., H. Kurogi, H. Kunitake, K. Nagano, T. Yabuya, K. Yamashita and H. Komatsu. 2005b. Evaluation of reproductive functions in a haploid pummelo by crossing with several diploid citrus cultivars. J. Japan. Soc. Hort. Sci. 74: 281-288.

Yamamoto, M., A. A. Abkenar, R. Matsumoto, H. Nesumi, T. Yoshida, T. Kuniga, T. Kubo and S. Tominaga. 2007. CMA banding pattern of chromosomes in major Citrus species. J. Japan. Soc. Hort. Sci. 76: 36-40.

Yamamoto, M., A. A. Abkenar, R. Matsumoto, T. Kubo and S. Tominaga. 2008. CMA staining analysis of chromosomes in several species of Aurantioideae. Genet. Res. Crop Evol. 55: $1167-1173$.

Yamamoto, M. and S. Tominaga. 2003. High chromosomal variability of mandarins (Citrus spp.) revealed by CMA banding. Euphytica 129: 267-274.

Yamashita, K., Y. Takatori and Y. Tashiro. 2005. Chromosomal location of a pollen fertility-restoring gene, $R f$, for CMS in Japanese bunching onion (Allium fistulosum L.) possessing the cytoplasm of A.galanthum Kar. et Kir. Revealed by genomic in situ hybridization. Theor. Appl. Genet. 111: 1522.

Yao, J. L., D. Cohen and R. E. Rowland. 1994. Plastid DNA inheritance and plastome-genome incompatibility in interspecific hybrids of Zantedeschia (Aranceae). Theor. Appl. Genet. 88: 255-260.

Zhou, A. and G. Xia. 2005. Introgression of the Haynaldia villosa genome into $\gamma$-ray-induced asymmetric hybrids of wheat. Plant Cell Rep. 24: 289-296. 